Relations industrielles

Industrial Relations

\title{
Organized Business in France, by Henry W. Ehrmann. Princeton, N.J.: Princeton University Press, 1957, 514 pp. \$7.50.
}

\section{Gérard Dion}

Volume 14, numéro 2, avril 1959

URI : https://id.erudit.org/iderudit/1022324ar

DOI : https://doi.org/10.7202/1022324ar

Aller au sommaire du numéro

Éditeur(s)

Département des relations industrielles de l’Université Laval

ISSN

0034-379X (imprimé)

1703-8138 (numérique)

Découvrir la revue

Citer ce compte rendu

Dion, G. (1959). Compte rendu de [Organized Business in France, by Henry W.

Ehrmann. Princeton, N.J.: Princeton University Press, 1957, 514 pp. \$7.50.]

Relations industrielles / Industrial Relations, 14(2), 302-303.

https://doi.org/10.7202/1022324ar

Tous droits réservés (C Département des relations industrielles de l’Université Laval, 1959
Ce document est protégé par la loi sur le droit d'auteur. L’utilisation des services d'Érudit (y compris la reproduction) est assujettie à sa politique d'utilisation que vous pouvez consulter en ligne.

https://apropos.erudit.org/fr/usagers/politique-dutilisation/ 


\section{RECENSIONS - BOOK REVIEWS}

Organized Business in France, by Henry W. Ehrmann. Princeton, N.J.: Princeton University Press, 1957, 514 pp. $\$ 7.50$.

La littérature sur le syndicalisme ouvrier est très abondante. Il n'en est pas de même en ce qui regarde les organisations patronales. Et c'est aisé à comprendre quand on considère que la multiplicité des groupes, le genre d'action qu'ils déploient tant dans le domaine économique que politique rendent les analyses extrêmement difficiles.

Quand l'auteur a entrepris cette étude sur les groupements patronaux français, il avait l'intention de préparer un ouvrage qui ferait le pendant d'un autre qu'il avait publié sur l'histoire du mouvement ouvrier français. Il voulait rechercher quels effets la réaction des employeurs avait eus sur la politique de relations du travail au cours de la période qui a marqué le déclin de la troisième République, durant le régime de Vichy, et le commencement de la quatrième République. Ses recherches l'ont conduit beaucoup plus loin.

L'étude de groupes de pression ne peut se faire sans connaître les autres facteurs qui influencent toute la vie publique, le contexte culturel, les idéologies économiques et politiques, etc. L'auteur se garde de présenter son ouvrage comme une analyse satisfaisante du comportement des employeurs français ou une description du capitalisme en ce pays au milieu du vingtième siècle. Il ne prétend pas non plus voulair faire une analyse comparative avec les autres pays. Cependant, avec sa formation, son orientation d'esprit et ses schèmes de références, on trouve dans son étude les éléments qui permettent aux lecteurs familiers avec la réalité américaine de saisir eux-mêmes ce que l'auteur suggère.

L'ouvrage est divisé en quatre parties. La première couvre la période du Front Populaire à la Libération. La seconde porte sur la structure et les activités des organisations patronales actuelles.
La troisième étudie leurs attitudes et politiques dans les problèmes économiques et les relations industrielles. La quatrième est la conclusion.

L'auteur est très bien informé. Il ne s'est pas contenté d'utiliser une abondante documentation et de la critiquer, mais il a interviewé un nombre considérable d'employeurs, de dirigeants d'associations patronales et d'officiers supérieurs du gouvernement. C'est une étude exhaustive, honnête. Il ne craint pas d'exprimer franchement ce qui lui semble des contradictions dans l'attitude des employeurs, d'évaluer l'efficacité de leurs attitudes.

"At present the ambivalent position in which the leadership of organized business is caught, explains the often noted contradictions in its behavious. Frequently a gap between announced principles and day-to-day activities exposes the movement to ridicule. Individual firms or their associations are taken to task by other sectors of the employers' movement or by the CNPF for their sluggishness, but when general economic conditions are considered unsatisfactory, business still blames, as it always has, labor, rival nations, and governmental policies for all and every difficulty."

Tout en manifestant beaucoup d'amitié et d'admiration pour le peuple français, ses conclusions ne sont pas trop optimistes.

"Succeeding cabinets have looked in vain to an informed public for the support of progressive polioies. Without a new public spirit most institutional and economic reforms may prove as futile as many of them have in the past. The welfare state which France has long since become, cannot function properly as long as a large number of Frenchmen in all social categories deny its philosophy and flout its rules. To bring about widespread acceptance of the general premises on which state and society are to operate is everywhere a long and complicated process. In Fran- 
ce prognostics of suocess or failure in solving a crisis situation have at all times been particularly hazardous because of the contradiotions and surprising reversals of public and private attitudes."

\section{GÉrard Dion}

Brève histoire du syndicalisme ouvrier au Canada, Ls-Laurent Hardy, Coll. \& Les voix ». Les Editions de l'Hexagone, Montréal 1958, 155 pp.

Les ouvrages en langue française sur le syndicalisme ouvrier au Canada sont rares. En dehors des essais de Charpentier sur le syndicalisme catholique, du volume de J.-P. Després \& Le mouvement ouvrier canadien » publié par notre département chez Fides en 1947, on ne trouve que des brochures, des articles de revues, (particulièrement dans Relations Industrielles) qui envisagent l'un ou l'autre aspect de cette question. Le titre du volume de M. Hardy est exact. C'est une brève synthèse que présente l'auteur. Il s'est servi des études déjà publiées en langue anglaise et a eu accès aux archives de la CTCC. Son mérite n'est pas tant l'originalité ni l'analyse des causes ou circonstances qui ont influencé l'évolution du syndicalisme, que d'être une synthèse qui embrasse tous les événements jusqu'à aujourd'hui. L'auteur, avec raison, s'étend beaucoup plus longuement sur la CTCC que les autres centrales. Il comble ainsi une lacune que l'on pouvait déplorer chez Logan, "Trade Union in Canada" (MacMillan, 1948) et même, à un degré moindre cependant, chez Jamieson, "Industrial Relations in Canada" (MacMillan, 1957). Une véritable histoire du syndicalisme au Canada - et surtout dans la province de Québec, - reste à faire. Cependant nous croyons que M. Hardy a rendu à tous un grand service en publiant cette synthèse. Même les syndiqués y apprendront quelque chose.

\section{GÉRARD Dion}

The Appraisal Interview. By N.R.F. Maier, New York: John Wiley \& Sons, 1958, 246 pp.

Ce volume a pour but d'étudier la relation de cause à effet qui existe dans une situation d'entrevue dont le but est d'évaluer la performance de l'employé.
Depuis de nombreuses années Maier s'est voué à la recherche dans le domaine des relations humaines. Il s'est surtout manifesté dans le développement et l'entraînement du personnel de la gérance. Il a constaté que l'entrevue d'évaluation (appraisal interview) est utilisée pour développer le personnel administratif, mais que les résultats obtenus sont superficiels ou nuls. Pourquoi? Parce qu'en général on a voulu trop accomplir avec cette méthode et que ce faisant on n'arrivait à aucun résultat pratique. Plusieurs objectifs que l'on tentait d'atteindre d'un seul coup se sont montrés incompatibles. Placé dans une situation confuse, l'employé considérait l'évaluation comme injuste et arbitraire.

Maier démontre qu'en étudiant les objectifs à atteindre on peut utiliser trois méthodes. Chacune d'elles est caractérisée par une entrevue qui demande une technique d'approche bien spécifique. Voici un résumé de chaque entrevue.

\section{1-L'entrevue d'évaluation directive: \\ (Tell and Sell Method)}

L'objectif initial de cette méthode est de communiquer objectivement à l'employé les résultats de l'évaluation. De plus, le patron doit faire accepter cette évaluation par l'employé et l'amener à suivre un programme d'amélioration prédéterminé. Parce qu'elle est simple, cette méthode est la plus utilisée. Heureusement Maier a tôt fait de démontrer qu'elle est inefficace ot même dangereuse pour la raison suivante: elle assume que l'employé acceptera tout et changera son comportement.

Toutefois si l'employé refuse l'évaluation, le patron devient agressif. La situation détériore rapidement soit en un conflit soit en une soumission passive de la part du subalterne. Incidemment les gains abtenus sont insignifiants comparativement aux pertes: perte d'intérêt pour le travail, relations tendues entre patron et employé. L'employé apprend à faire ce qui plaît au patron. Cette approche autoritaire et dominatrice, qui empêche l'employé d'exposer son point de vue, tue toute initiative et toute créativité. Ce sont les considérations émotives et subjectives qui dominent dans l'entrevue d'évaluation directive. 